\title{
Amplification of the Evanescent Field of Free Electrons
}

\author{
J. K. So ${ }^{\dagger, *}$, J. Y. Ou ${ }^{\dagger}$, G. Adamo ${ }^{\dagger}$, K. F. MacDonald ${ }^{\dagger}$, F. J. Garcia de Abajo ${ }^{\ddagger \dagger}$, and N. I. Zheludev ${ }^{\dagger}$ \\ ${ }^{\prime}$ Optoelectronics Research Centre, University of Southampton, SO17 1BJ, United Kingdom \\ \$ Instituto de Óptica - CSIC, Serrano 121, 28006 Madrid, Spain \\ *js1m10@orc.soton.ac.uk
}

\begin{abstract}
A nanoscale plasmonic metal film can amplify free-electron evanescent fields, leading to strongly enhanced light emission via Smith-Purcell effect through a mechanism analogous to the 'poor-man's superlens' for optical evanescent field enhancement.

OCIS codes: (240.6680) Surface plasmons; (230.6080) Optical devices, Sources
\end{abstract}

We demonstrate that a layer of silver a few tens of nanometers thick can amplify the evanescent field of free electrons in the same way that the plasmonic metal acts on the evanescent field of light. The enhancement of the evanescent field of free electrons is studied by analyzing the far-field emission from nano-gratings on the tips of optical fibers in a scanning electron microscope (Fig. 1) and through numerical simulations showing an order of magnitude increase in Smith-Purcell radiation. The enhancement exhibited here for Smith-Purcell radiation may readily be applied to other forms of electron-beam-induced light emission.

The electromagnetic energy of the evanescent field of free electrons exists in the form of evanescent waves and can be decoupled into light only when they are in close proximity to a slow-wave medium or optical inhomogeneity. The former gives rise to what is famously known as Cerenkov radiation, the latter to diffraction or Smith-Purcell radiation [1]. Since the exponential decay rate of such evanescent waves is proportional to the ratio of the frequency of the electromagnetic field to the velocity of the particle, high velocity, relativistic particles are more efficient in generating light in the optical part of the spectrum. Here, rather than increasing the energy of particles to achieve higher levels of conversion, we utilize a thin silver layer to amplify the rapidly decaying evanescent waves of nonrelativistic free electrons.

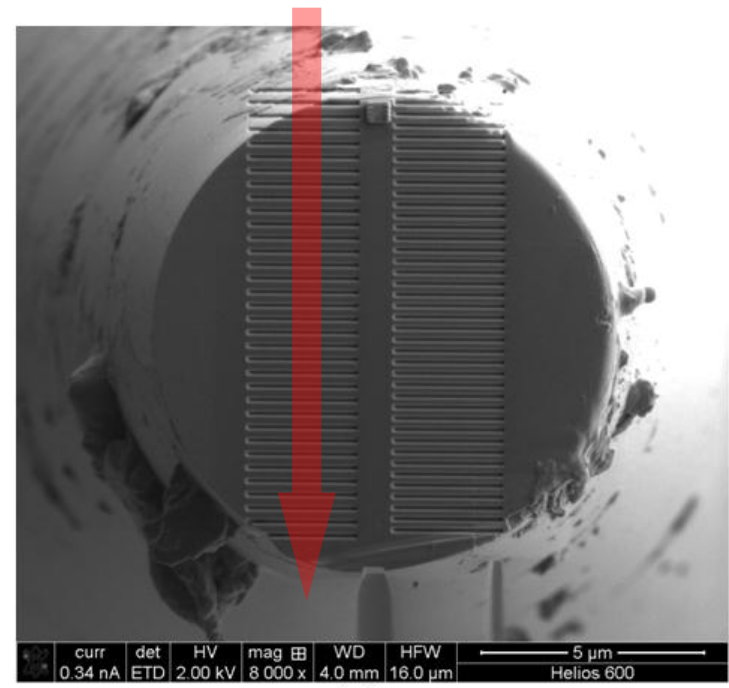

(a)

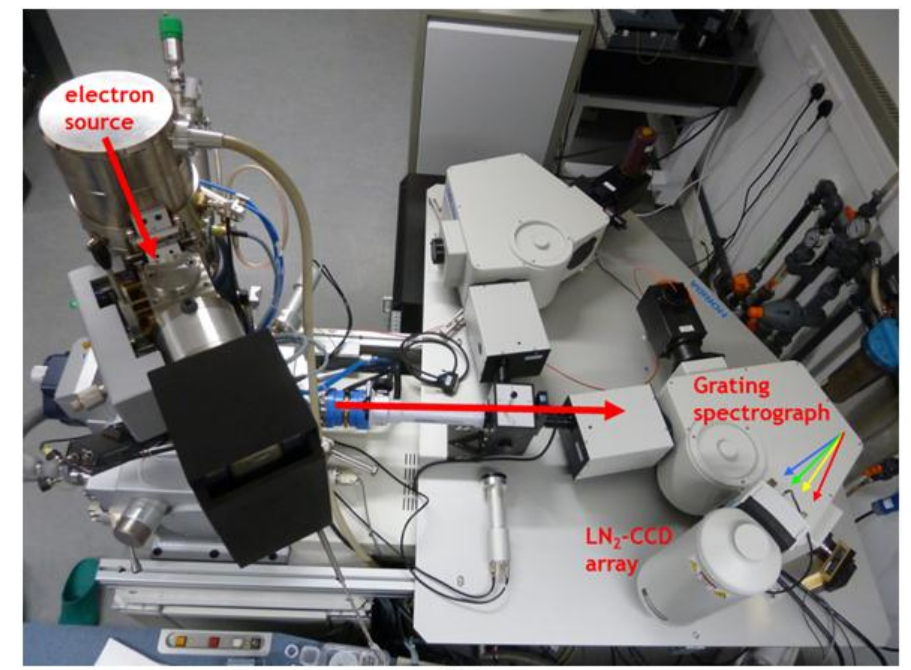

(b)

Fig. 1: (a) Electron microscope image showing nano-gratings fabricated on a tapered fiber tip [electrons pass over the grating as indicated by the arrow], Smith-Purcell radiation is collected via the fiber; (b) Experimental configuration comprising a scanning electron microscope and a UV-VIS spectrometer equipped with a liquid-nitrogen cooled CCD detector.

In analogy to the 'poor-man's superlens' where the evanescent component of light from an object is restored by a thin silver layer to beat the diffraction limit [2], we consider a thin silver layer for the amplification of the free electron evanescent field before it is decoupled into light by a nano-grating. The period of the grating is chosen so that the Smith-Purcell emission wavelength in the surface-normal direction coincides with the plasmonic resonance 
of the silver layer. Nano-gratings are manufactured by focused ion-beam milling on the end faces of optical fibers as shown in Fig. 1a. The fiber end face is aligned parallel to the electron beam trajectory in a scanning electron microscope and the light generated from the nano-structure is collected via the fiber and transported to a UV-VIS spectrometer equipped with a liquid-nitrogen-cooled CCD array as shown in Fig. 1b.

The interactions between nano-structures and free electron beams have also been studied numerically using the particle-in-cell (PIC) methodology, assuming a Gaussian electron bunch traversing the structure as shown in Fig. 2a. The electron bunch was modeled with a lateral width equal to one quarter of that of the grating to ensure coherent emission in the spectral range considered. The presence of a silver layer provides for the excitation of surface plasmons satisfying phase-velocity synchronism with the electrons, and leads to the amplification of the electrons' evanescent field.

(a)

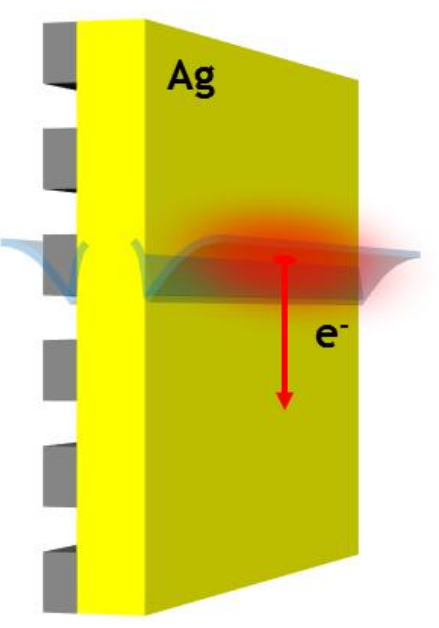

(b)

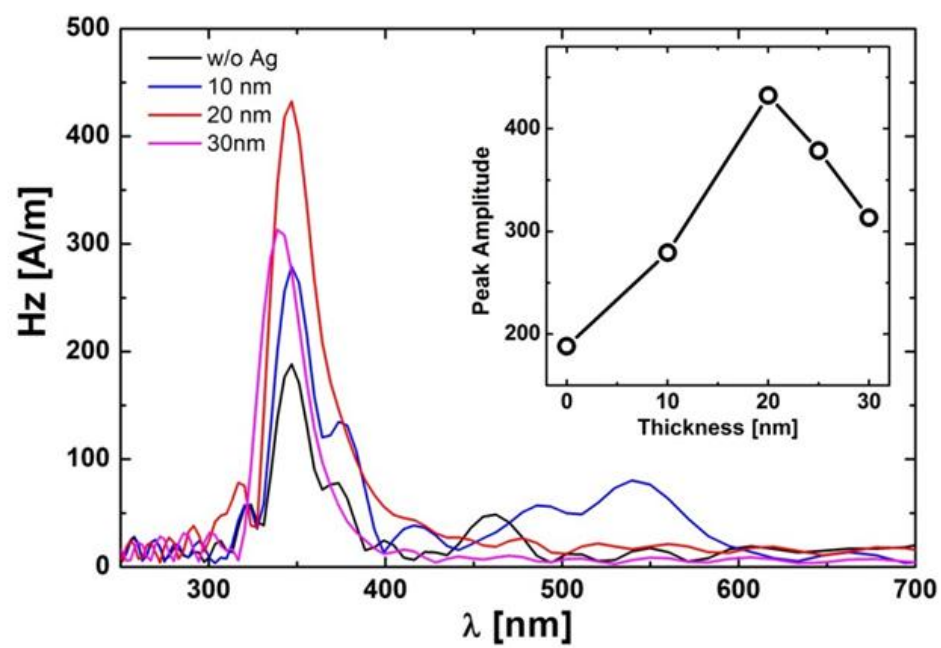

Fig. 2: (a) Schematic model of a silver layer on a nano-grating and free electrons as considered in particle-in-cell simulations;

(b) Spectra of light emission induced by $40 \mathrm{keV}$ electrons for various thicknesses of the silver layer. The inset shows the strength of the emission peak at $\sim 350 \mathrm{~nm}$ as a function of silver layer thickness, revealing a maximum at $\sim 20 \mathrm{~nm}$.

Free electrons with an energy of $40 \mathrm{keV}$ traversing a nano-grating with a period of $130 \mathrm{~nm}$ emit first-order Smith-Purcell radiation at around $350 \mathrm{~nm}$ as shown by the spectrum in Fig. 2b. As a silver layer is introduced, the magnitude of the evanescent field at the location of the grating is enhanced and it is this enhanced evanescent field that is decoupled by the grating. As a result, Smith-Purcell radiation is enhanced. The extent of this enhancement depends on the thickness of the silver layer but losses also increase with thickness - the optimum thickness is found to be $\sim 20 \mathrm{~nm}$ (see Fig. 2b).

In summary, we show that the emission of Smith-Purcell radiation can be amplified by enhancing the evanescent field of fast-moving electrons before it reaches the grating at which decoupling into far-field light occurs. The evanescent field enhancement is achieved by introducing an intermediate silver layer supporting surface plasmons in the spectral range of Smith-Purcell radiation for the electron kinetic energy.

[1] S. J. Smith and E. M. Purcell, "Visible Light from Localized Surface Charges Moving across a Grating”, Phys. Rev. 92, 1069 (1953).

[2] J. B. Pendry, "Negative Refraction Makes a Perfect Lens", Phys. Rev. Lett. 85, 3966 (2000). 